Other

Suhaila Idayu Abdul Halim, Chin Han Chan* and Jan Apotheker

\title{
Basics of teaching electrochemical impedance spectroscopy of electrolytes for ion-rechargeable batteries - part 1: a good practice on estimation of bulk resistance of solid polymer electrolytes
}

https://doi.org/10.1515/cti-2020-0011

Received June 13, 2020; accepted February 19, 2021; published online March 8, 2021

\begin{abstract}
In this publication, we present the basic to characterize the electrical properties of electrolytes that are widely used in ion-rechargeable batteries using electrochemical impedance spectroscopy (EIS). This simplified yet insightful background provided may be used for educational purposes, especially for beginners or young researchers for both undergraduate and postgraduate students. We start with introduction of electrolytes and electrochemical impedance spectroscopy (EIS) instrumentation, following with the step-by-step guidelines using three different procedures to estimate the bulk resistance $\left(R_{\mathrm{b}}\right)$ of the electrolytes, which is inversely proportional to the conductivity $\left(\sigma_{\mathrm{DC}}\right)$ of the materials $\left(R_{\mathrm{b}} \propto 1 / \sigma_{\mathrm{DC}}\right)$. Several examples and exercises on estimation of quantity $R_{\mathrm{b}}$ are supplemented for educational purposes. Comparison was made on estimation of $R_{\mathrm{b}}$ using manual graphical procedures, mathematical regression procedures using commercial graphical software and equivalent circuit fitting procedures using exclusive EIS software. The results suggest that the manual graphical technique may serves as a useful approach for beginners before venturing to exclusive software. Besides, the instructors may use the procedures to coach the users to extract reliable and reproducible data before data interpretation. Lastly, the phenomenological approach on dielectric relaxation for solid polymer electrolytes [poly(ethylene oxide) (PEO) + lithium salt] and non-solid polymer electrolytes [poly(methyl acrylate) (PMA) + lithium salt], in the classic sense will be addressed in terms of impedance $\left(Z^{\star}\right)$, permittivity $\left(\varepsilon^{\star}\right)$, tangent loss $(\tan \delta)$, modulus $\left(M^{\star}\right)$ and conductivity $\left(\sigma^{\star}\right)$ spectra in Part 2.
\end{abstract}

Keywords: bulk resistance; electrochemical impedance spectroscopy; impedance properties; solid polymer electrolytes.

This is one of the instructor's notes based on the short course presented at the 25th POLYCHAR 2017 World Forum on Advanced Materials, Kuala Lumpur, Malaysia, October 9-13, 2017. The power point slides can be accessed via https://iupac.org/wp-content/ uploads/2017/12/IUPAC_PolymEdu_Shortcourse_1ppt_ChinHanChan.pdf and the

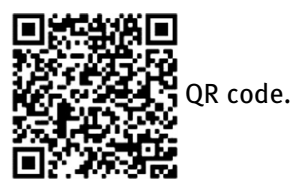

*Corresponding author: Chin Han Chan, Faculty of Applied Sciences, Universiti Teknologi MARA, 40450 Shah Alam, Selangor, Malaysia, E-mail: cchan_25@yahoo.com.sg. https://orcid.org/0000-0003-0990-0155

Suhaila Idayu Abdul Halim, Faculty of Applied Sciences, Universiti Teknologi MARA, 40450 Shah Alam, Selangor, Malaysia Jan Apotheker, Faculty of Sciences and Engineering, University of Groningen, 9747 AG, Groningen, Netherlands

Ә Open Access. ( 2021 Suhaila Idayu Abdul Halim et al., published by De Gruyter. $(\Subset)$ BY This work is licensed under the Creative Commons Attribution 4.0 International License. 


\section{Introduction}

\section{Electrolytes and batteries}

Electrolyte is an electrically conducting solution when a substance (e.g., ionic compound, dipolar entity etc.) dissolve in a polar matrix (e.g., polar solvent). An electrolyte is known as a medium that is capable for transporting electrical charges under the action of electric field, which owing from the movement of charged molecules. An electrolyte commonly serves as a medium of a (rechargeable) battery, which allows the flow of charge entities from one electrode to another electrode. A battery cell (refer Figure 1) is an electrochemical cell that is comprised of an electrolyte sandwiched in between two electrodes, named as anode and cathode. When a (rechargeable) battery is in the discharge state (Galvanic cell) connected to an external electric circuit, the electrons will spontaneously flow from the more negative electrode potential to the more positive electrode potential through the external circuit as a result of the spontaneous redox reaction at the respective electrodes. This implies oxidation occurs at negative electrode (anode), the reduction occurs at the positive electrode (cathode), and charged entities transport between the electrodes by passing through electrolyte for generation of electric current (Plante, 1884; Voss, 1990). However, the redox reaction can be reversed by means of applying an external electric potential (voltage) to produce complementary redox reaction at the electrodes. This configuration is a non-spontaneous and energy-dependent process, which can be observed in the rechargeable battery (secondary) that is in the charged state (electrolytic cell) (Learn Engineering, 2019; Ramström, 2019).

Electrolytes can be classified in the form of liquid, gel and solid. Liquid electrolyte is known for its excellent characteristic as a good electric conductor. For a long time, liquid electrolytes have been widely used in the Li-ion (rechargeable) battery owing to their superior electrical properties (Baril, Michot, \& Armand, 1997; Klinklai et al., 2006). A liquid electrolyte has the configuration of a strong electrolyte, as the ionic compounds dissociate into cations and anions. However, apart from being a highly conductive compounds, liquid electrolyte possesses major drawbacks that greatly limits its further development and wider application, i.e., high in toxicity, explosive, flammable, unstable electrochemical performance and environmentally harmful. Highly flammable electrolyte might be responsible for the recent catastrophic battery explosions (Kong, Li, Jiang, \& Pecht, 2018; Mackanic et al., 2019; Manthiram, Yu, \& Wang, 2017). Gel electrolytes are formulated with the combined features of both solid and liquid electrolytes have gained attentions for the past decades for replacement of liquid electrolytes (Baskoro, Wong, \& Yen, 2019; Ngai, Ramesh, Ramesh, \& Juan, 2016;

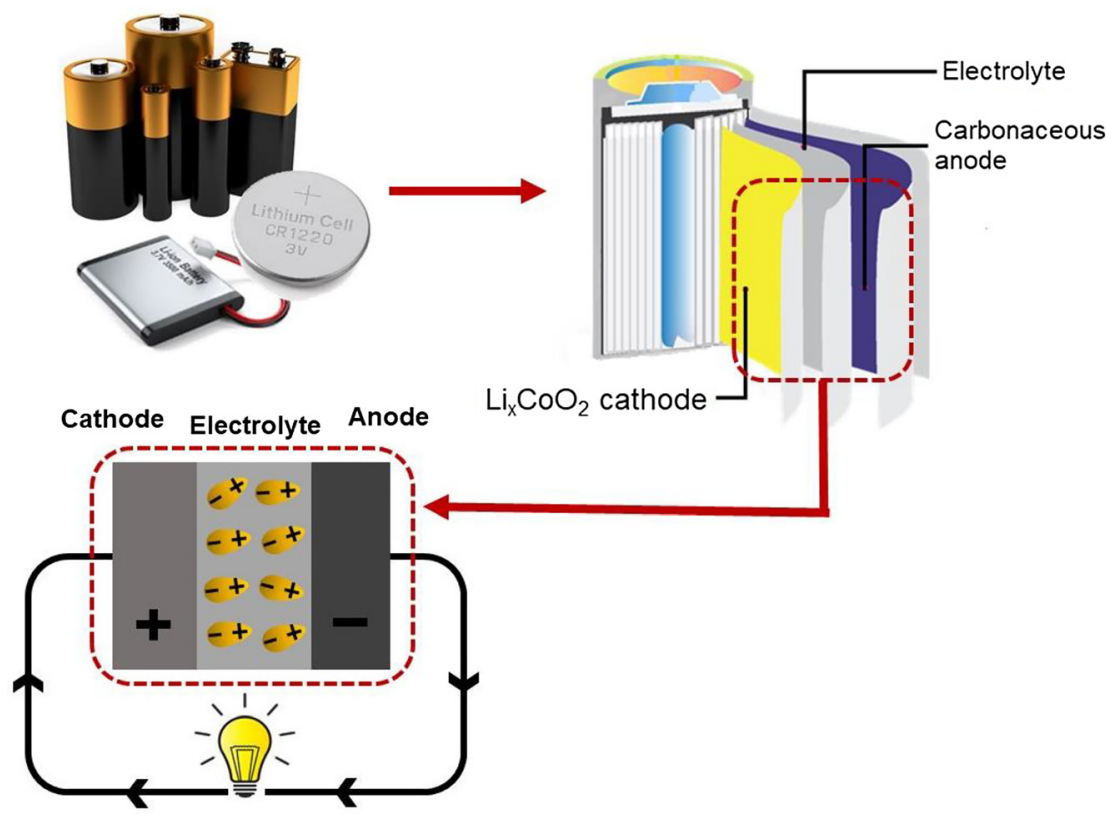

Figure 1: Illustration of basic configuration of a lithium ion (rechargeable) battery (Li-ion battery) in the discharged form. Electrons flow through the circuit as a result of the spontaneous redox reactions at the respective electrodes. 


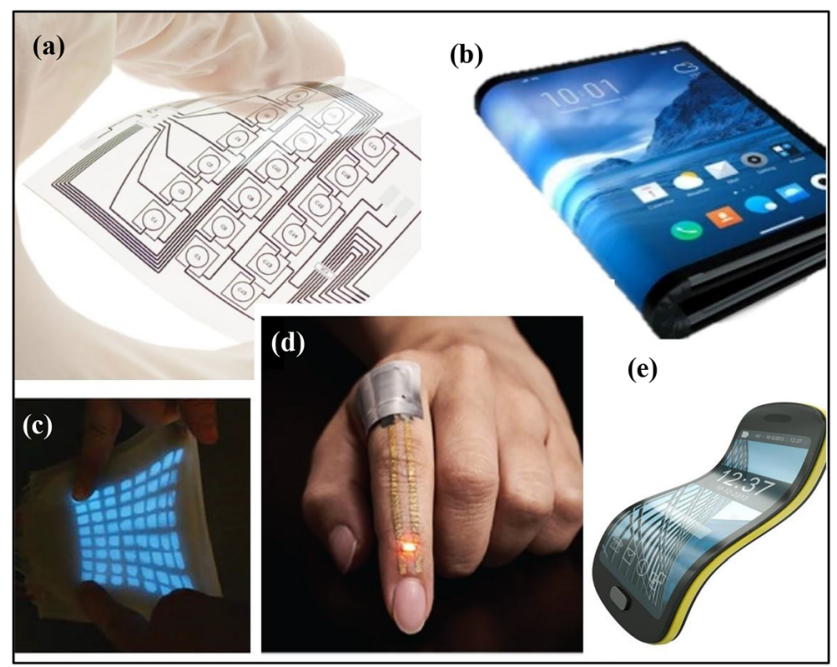

Figure 2: Prototypes of flexible or soft electronics. (a) Hybrid-integrated flexible electronic system, (b and e) future perspective of mobile electronic devices, (c) stretchable display for optical and tactile sensing, reproduced with permission from (Larson et al., 2016), copyright 2016, American Association for the Advancement of Science, and (d) on-skin electronic sensor for health monitoring, reproduced with permission from (Miyamoto et al., 2017), copyright 2017, Springer Nature.

Stephan, 2006). However, the safety concerns still arise (e.g., volatility, flammability etc.) although the operational stability of gel electrolytes is pretty decent (Kong et al., 2018). Therefore, many researchers have conducted extensive studies since 70s to overcome the limitation of liquid and gel electrolytes for modern Li-ion battery applications by designing and inventing solid electrolytes with high ionic conductivity and more distinctive features as compared to the other two electrolytes. Solid electrolytes have been seen as potential materials with high energy density, high power, high durability, high flexibility and non-flammable for energy storage applications.

Solid polymer electrolyte (SPE) is formed by dissolution of low-molar-mass electrolyte (e.g., lithium salt, sodium salt etc.) in polymer matrix, usually the polar polymers [e.g., poly(ethylene oxide) (PEO) (Chan \& Kammer, 2015; Pratap \& Chandra, 2013), poly(propylene oxide) (PPO) (Su, Wang, \& Liu, 2002; Zhang \& Chen, 2004), poly(methyl methacrylate) (PMMA) (Raja, Sharma, \& Rao, 2004; Ramesh \& Bing, 2012), epoxidized natural rubber (ENR) (Hussin, Harun, \& Chan, 2017; Mohd Yusoff, Sim, Chan, Hashifudin, \& Kammer, 2013) etc.] are noted. Special attention has placed on SPE for Li-ion rechargeable batteries due to the theoretical specific energy density at $4.2 \mathrm{~V}$ can be achieved up to $380-460 \mathrm{Wh} \mathrm{kg}^{-1}$ especially when the SPE is combined with the existing large-capacity cathode and anode (Li, Chen, Fan, Kong, \& Lu, 2016), apart from being flexible, light in weight, and non-toxic etc. in some cases. Up to date, SPE is considered as a highly potential material for the next generation high-energy rechargeable batteries. Credits to the ever-increasing demand on flexible energy storage and power applications, we will able to see the invention of flexible and powerful SPE as a medium of rechargeable battery in the near future and the revolution is expected to emerge rapidly for the next 20-30 years (Li et al., 2016; Liu et al., 2019; Mackanic et al., 2019; Manthiram et al., 2017; Ramström, 2019). Figure 2 displays the several examples of advanced prototypes for flexible or soft electronics embedded with energy storage devices (Larson et al., 2016; Miyamoto et al., 2017) for potential commercial applications.

One of the standard requirements of SPEs for the rechargeable battery is the ionic conductivity $\left(\sigma_{\mathrm{DC}}\right)$ should be at least $\sim 10^{-3} \mathrm{~S} \mathrm{~cm}^{-1}$ at room temperature (Li et al., 2016; Liu et al., 2019; Lopez, Mackanic, Cui, \& Bao, 2019; Mackanic et al., 2019). The estimation of $\sigma_{\mathrm{DC}}$ is calculated from the bulk resistance $\left(R_{\mathrm{b}}\right)$ using EIS in this study, where $\sigma_{\mathrm{DC}}=L /\left(A \cdot R_{\mathrm{b}}\right)$. Quantities $L$ and $A$ denote the thickness of the sample and surface area of the sample film, which contact with the electrodes, respectively. Since quantity $\sigma_{\mathrm{DC}}$ is crucial for commercial applications of the SPEs, hence in this presentation, a good practice on the estimation of $R_{\mathrm{b}}$ will be highlighted. Three Supplementary Files (i.e., S1 - A step-by-step guideline to estimate bulk resistance $\left(R_{\mathrm{b}}\right)$ using mathematical regression with commercial graphical software, $\mathbf{S 2}$ - A step-by-step guideline to estimate $R_{\mathrm{b}}$ using manual graphical approach and $\mathbf{S} 3$ - A step-by-step guideline to estimate $R_{\mathrm{b}}$ using exclusive EIS software) are provided separately for educational purposes. Consistency in data extraction is crucial in order to obtain a precise and reproducible data. 
Secondly, two systems are used (SPE; binary poly(ethylene oxide) (PEO) + salt and non-SPE; binary poly(methyl acrylate) (PMA) + salt) in the classic sense to show the phenomenological dielectric response of the imaginary part of impedance $\left(Z^{\prime \prime}\right)$, permittivity $\left(\varepsilon^{\star}\right)$, tangent loss $(\tan \delta)$, imaginary part of the electric modulus $\left(M^{\prime \prime}\right)$ and conductivity $\left(\sigma^{\star}\right)$, which will be discussed in a more detailed manner in the next article as Part 2. The presentation on EIS has been kept from mathematics and electrical theory as much as possible for beginners.

\section{Basics - electrolytes}

An electrolyte may be classified as a strong or a weak electrolyte. A strong electrolyte is when the ionic compounds dissociate completely into free ions (e.g., cation and anion) in a polar matrix [c.f. Figure 3(a)]. If only a relatively small fraction of the ionic compound dissociates into free ions or exist as separated ion pairs or contact ion pairs in a polar matrix, it is classified as a weak electrolyte [c.f. Figure 3(b) and (c)]. The degree of dissociation of the ions in the polar solvent is mainly governed by the strength of electrostatic interaction between the charged molecules along with the electrolyte concentration $(c)$. The measurement of the strength of the electrostatic attraction of the ion pair in a medium (polar solvent) is called the Bjerrum length $\left(\lambda_{\mathrm{B}}\right)$. It is defined as the distance at which the electrostatic interaction (energy) of the ion pair is equal to the thermal energy $k_{\mathrm{B}} T$,

$$
\lambda_{\mathrm{B}}=\frac{e^{2}}{4 \pi \epsilon_{\mathrm{o}} \epsilon k_{\mathrm{B}} T}
$$

where $e$ is the absolute value of the electronic charge, $\varepsilon_{\mathrm{o}}$ is the permittivity of the vacuum and $\varepsilon$ is the dielectric constant of the medium. Equation (1) tells the strength of the interaction $\lambda_{\mathrm{B}}$ is highly dependent on the dielectric constant of the medium, the valences of the ions as well as the $c$. Ionic compounds in a medium with higher dielectric constant will have shorter $\lambda_{\mathrm{B}}$ and have significant volume of free ions due to the higher solvation effect (Adar, Markovich, \& Andelman, 2017; Lee, Perez-Martinez, Smith, \& Perkin, 2017). The quantity of $\lambda_{\mathrm{B}}$ could be important to deliberate the electric potential of the solution. Figure 3(a) shows the example of aqueous sodium chloride $(\mathrm{NaCl})$, where the $\lambda_{\mathrm{B}}$ is shorter than the distance of separation of the charged molecules, and this phenomenon only can be seen in solution with $c \rightarrow 0$ (Fuoss, Onsager, \& Skinner, 1965). The ion pairs (charged molecules) possess stronger interaction if the distance of the ion pair is shorter than $\lambda_{\mathrm{B}}$

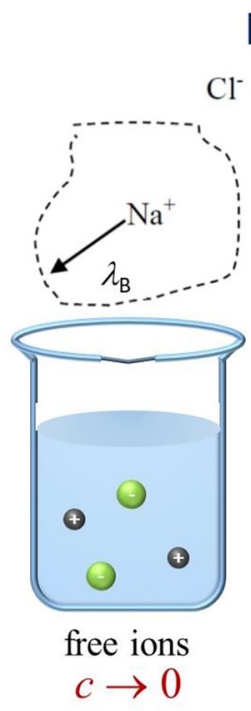

(a)

$\mathrm{Cl}^{-}$

\section{$\mathrm{NaCl} \leftrightarrow \mathrm{Na}^{+}+\mathrm{Cl}^{-}$}

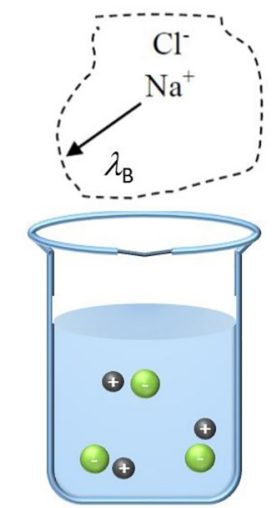

separated ion pairs

(b)

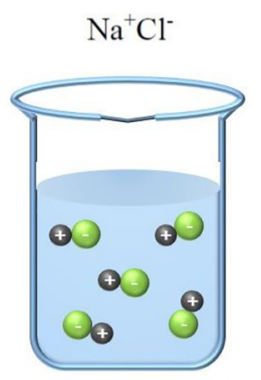

contact ion pairs

(c)
Figure 3: Three possible ion configurations in a solution using the example of aqueous sodium chloride $(\mathrm{NaCl})$ (Fuoss, 1965). Quantity $c$ denotes as concentration of the electrolyte. 
for higher $c$ and vice versa for $c \rightarrow 0$, that indicates to the interaction of the ion pairs in the solvent is concentration dependent (Muthukumar, 2012).

SPE is known as dielectric material because it has the capability to store electrical charges and can only be polarized when placed under an action of external electric field. There will be orientation of the dipolar entities (i.e., positive and negative charges) in the dielectric material to the oppositely charged poles when induced by an external electric field. It turns out that the orientation of dipolar entities makes one side of the dielectric material somewhat positive and the opposite side somewhat negative. A good dielectric material can be easily polarized under an electric field. The dielectric properties of SPE can be described in macroscopic scale by employing such concepts as dielectric constant, permittivity and polarization.

\section{Basics - electrochemical impedance spectroscopy (EIS)}

A simple direct current (DC) method (refer to Figure 1) cannot be used to estimate the resistance of a dielectric material due to the polarization of charges taking place at the electrode-electrolyte interface or at the phase boundaries of the sample or the combination of both as in Equation (2),

$$
R \equiv \frac{V}{I}
$$

where resistance $(R)$ is directly proportional to input voltage $(V)$ and inversely proportional to output current $(I)$. Polarization effect can be avoided if the alternating current $(\mathrm{AC})$ is applied to the sample, where the impedance $\left(Z^{\star}\right)$ of the sample is measured. Impedance measurement or the response of a SPE using EIS is estimated by applying a low amplitude sinusoidal potential (AC voltage) over a range of frequencies to the sample. EIS measures the time response or the dielectric relaxation time of a dielectric, which enables the evaluation or decoupling of small-scale polarization and conductance mechanism at the electrode interface and within the electrolyte, respectively (to be discussed in Part 2).

Dielectric polarization arises from a finite displacement of dipoles in a steady and flowing electric field. As illustrated in Figure 4, when a dielectric material (in this case, the SPE) is placed in between two electrodes under an electric field, the randomly oriented dipolar entities will start to align towards the direction of the oppositely charged electrodes.

The alignment of dipolar entities under the action of an alternating electric field is called dielectric polarization and will display dielectric relaxation that is born by the interplay of its resistance and capacitance at a particular frequency. Dielectric relaxation can be seen as the alignment of dipolar entities facing the oppositely charged electrodes at a certain frequency. Normally, if a SPE has one dielectric relaxation time constant, $\tau=\left(\omega_{\max }^{Z^{\prime \prime}}\right)^{-1}$, the system is known to follow Debye relaxation (ideal case) [c.f. Figure 5(a)] (Debye, 1929; Debye \& Falkenhagen, 1928). However, most of the systems show deviation from ideal Debye relaxation,

(a)

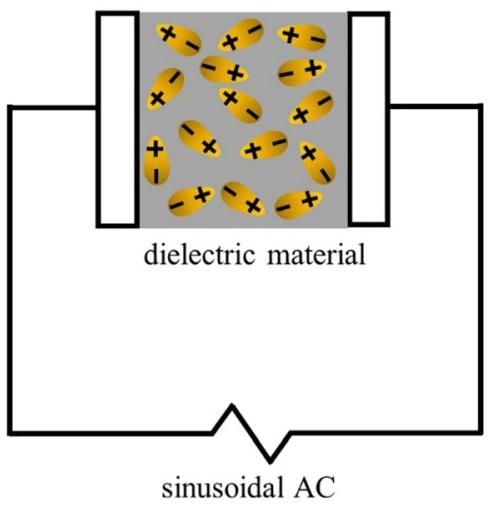

(b)

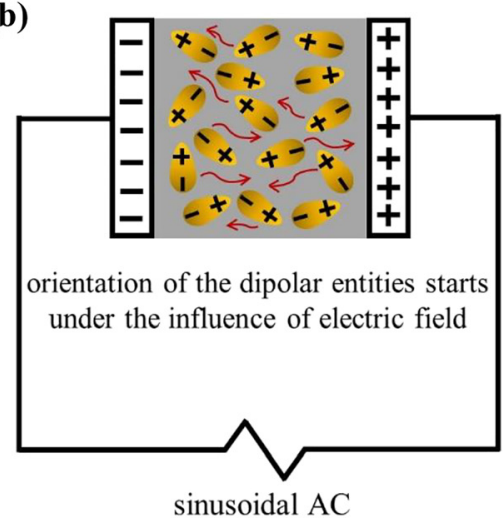

Figure 4: Orientation of dipolar entities of a dielectric material sandwiched in between the parallel-plate capacitor (blocking electrodes) under an action of electric field from (a) to (b). 
(a)

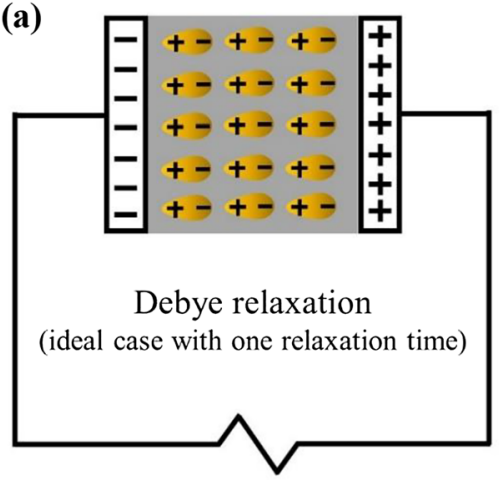

(b)

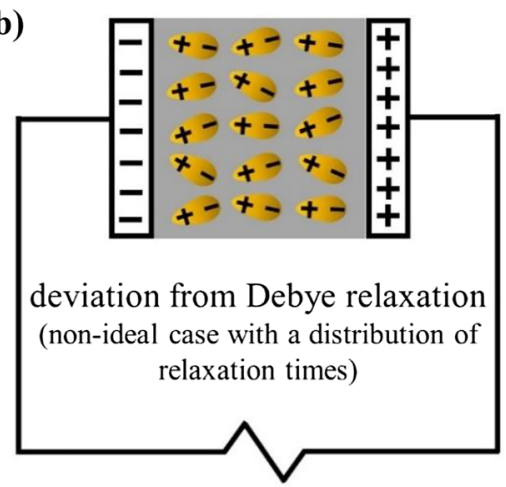

Figure 5: Orientation of dipolar entities of a dielectric material sandwiched in between the parallel-plate capacitor (blocking electrodes) connected to an EIS.

(a) following Debye response,

(b) deviation from Debye response.

Table 1: Characteristics of PEO and PMA polymer electrolytes.

\begin{tabular}{lrlrr}
\hline Characteristics & Molar mass $\left(\mathbf{g ~ m o l}^{-1}\right)$ & $W_{\mathbf{s}}{ }^{\mathrm{a}}$ (wt. \%) & $\boldsymbol{T}_{\mathbf{g}}{ }^{\mathrm{b}}\left({ }^{\circ} \mathbf{C}\right)$ & $\boldsymbol{T}_{\mathbf{m}}{ }^{\mathrm{C}}\left({ }^{\circ} \mathbf{C}\right)$ \\
\hline PEO & 300,000 & 0.5 and 11 wt. \% & -53 & 65 \\
PMA & 40,000 & 2 and 11 wt. \% & 13 & - \\
\hline
\end{tabular}

${ }^{\mathrm{a}}$ Salt content estimated from percentage of mass of salt/(mass of polymer + salt). ${ }^{\mathrm{b}}$ Glass transition temperature estimated using differential scanning calorimetry (Abdul Halim, Chan, \& Kammer, 2019). ${ }^{\mathrm{C}}$ Melting temperature estimated using differential scanning calorimetry (Halim et al., 2017).

due to heterogeneity of the systems, which leads to the distribution of dielectric relaxation times [c.f. Figure 5(b)].

EIS has been adopted to elucidate the electrical and dielectric relaxations in SPEs and non-SPEs. EIS data may be presented as Nyquist plot or Bode plot. For Nyquist plot, the frequency is hidden and for Bode plot, the frequency is explicit. These two plots are the key quantities of the interest in estimation of electric and dielectric properties of the SPEs and non-SPEs and their interfaces with electrodes (Chan \& Kammer, 2015, 2018, 2020). Here, the estimation of bulk resistance of SPEs and non-SPEs from Nyquist plots is discussed in Part 1 of this article and the other dielectric response of SPE and non-SPE in the frequency-dependant plot (or Bode plots) will be presented from the phenomenological point of view in the next article as Part 2.

\section{Experimental description}

The preparation procedures of SPEs used for this article were published elsewhere (i.e., dissolution of lithium perchlorate $\left(\mathrm{LiClO}_{4}\right)$ in poly(ethyne oxide) (PEO) and poly(methyl acrylate) (PMA), respectively) (Halim, Chan, \& Winie, 2017). Characteristics of the systems are given in Table 1.

Impedance measurement of each sample was estimated at $25^{\circ} \mathrm{C}$ using a Hioki $3532-50$ Hi Tester impedance analyzer (Hioki, Chubu, Japan) equipped with a computer for data collection over the frequency range from $50 \mathrm{~Hz}$ to $2 \mathrm{MHz}$. Two stainless steel electrodes with a diameter of $20 \mathrm{~mm}$ were used as the current collectors and the blocking electrodes for the ions. The sample was crimped in between the two blocking electrodes for measurement. The estimation of $R_{\mathrm{b}}$ values of the electrolytes will be explained in the discussions section. The values of $\sigma_{\mathrm{DC}}$ of the electrolytes can be estimated from $R_{\mathrm{b}}$ following equation of $\sigma_{\mathrm{DC}}=L /\left(A \cdot R_{\mathrm{b}}\right)$, where quantities $L$ and $A$ denote the thickness of the sample and surface area in touch with the two stainless steel disc electrodes, respectively. Values of $\sigma_{\mathrm{DC}}$ for SPEs and non-SPEs reported in this article were obtained from the averaging of three impedance analyses that were measured at three different spots of the sample with errors of $\sigma_{\mathrm{DC}}$ approximately at $10 \%$. 


\section{Discussions}

\section{Estimation of bulk resistance $\left(R_{\mathrm{b}}\right)$ from the Nyquist plot}

From the electrical point of view, SPE that consisting of ionic salt dissolves in a polymer, exhibits both resistive and capacitive behavior as reflected from the real $\left(Z^{\prime}\right)$ and imaginary $\left(Z^{\prime \prime}\right)$ parts of the complex impedance $\left(Z^{\star}\right)$, respectively, as illustrated in Figure 6. Figure 6(b) displays an equivalent circuit model of a SPE, where the resistance and capacitance are in parallel that can be distinguished using impedance spectroscopy over a range of frequencies (Randles, 1947). This equivalent circuit is one of the empirical models that was first introduced by Randles in order to compensate for double-layer capacitance phenomenon inspired by Frumkin and Grahame (Frumkin, 1940; Grahame, 1947). Double-layer capacitance also known as electrode polarization is a phenomenon that hinders the impedance measurements of most electrolytic systems due to the accumulation of charged ions at the respective opposite charged electrodes at low frequency region [as highlighted in red-dotted boxes shown in Figure 6(a) and (b)]. This phenomenon normally will result in the distribution of dielectric relaxations of the SPEs. However, this equivalent circuit is not unique to SPEs. Display of a good fit to experimental impedance data is not sufficient to validate a model. Consequently, we demonstrate an alternative to estimate $R_{\mathrm{b}}$ value using manual graphical method in the subsequent section before venturing to equivalent circuit fitting.

The data plots of $Z^{\prime}$ against $Z^{\prime \prime}$ of the impedance measured under AC over a range of frequencies, where the frequency is hidden, are illustrated in Figure 7. These plots are scientifically known as frequency dispersion spectrum or frequently called as Nyquist plot (Cole \& Cole, 1941). Each data point in the Nyquist plot represents the impedance of the electrolyte measured at a certain frequency. In fact, the plot is separated into frequency regions known as high frequency region and low frequency region as shown in Figure 7(a). The high frequency region is plotted toward the origin of the figure whereas the low frequency region is plotted outward from the origin of both $x$ - and $y$-axes.

The equivalent circuits are shown in Figure 7(a) and (b) in order to illustrate the common electrical elements used in the models used for fitting the impedance data as discussed in references (Madani, Schaltz, \& Kaer, 2019; Pei, Zhao, Yuan, Peng, \& Wu, 2018; Venkateswarlu \& Satyanarayana, 1998). We note here, the semicircle fitting in Figure 7(a) and (b) is not following the path of equivalent circuit fitting using commercial EIS software but following the mathematical regression approach to estimate the $R_{\mathrm{b}}$ value from a Nyquist plot with semicircle that was published in reference (Abdul Karim, Chan, \& Sim, 2017). The general procedures to estimate the $R_{\mathrm{b}}$ value using mathematical regression approach with commercial graphical software is enclosed in the Supplementary File $\mathbf{S 1}$ - A step-by-step guideline to estimate $R_{\mathrm{b}}$ using mathematical regression with commercial graphical software. The general idea is for a perfect semicircle (that following Debye response) centered at $\left(R_{\mathrm{b}} / 2,0\right), Z^{\prime}=Z^{\prime \prime}$. Hence, it can be seen

$$
\left(\mathrm{Z}^{\prime}\right)^{2}+\left(\mathrm{Z}^{\prime}\right)^{2}=\left(\frac{R_{\mathrm{b}}}{2}\right)^{2} \text { or }|Z|=\frac{R_{\mathrm{b}}}{2} \text { at } f_{\max }^{Z^{\prime \prime}}
$$

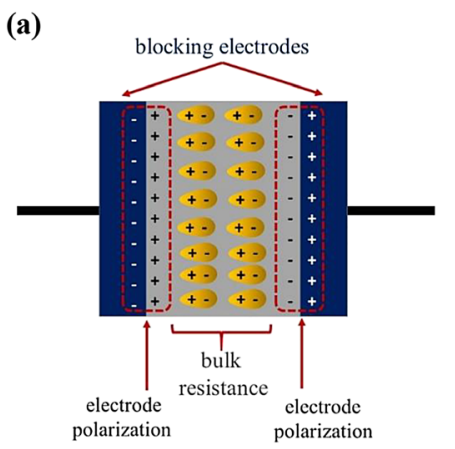

(b)

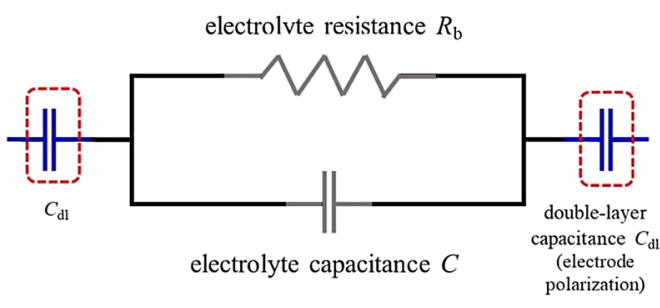

Figure 6: (a) Schematic drawing of SPE sandwiched in between two blocking electrodes following Debye response. (b) Equivalent circuit (model) of SPE sandwiched in between two blocking electrodes. 

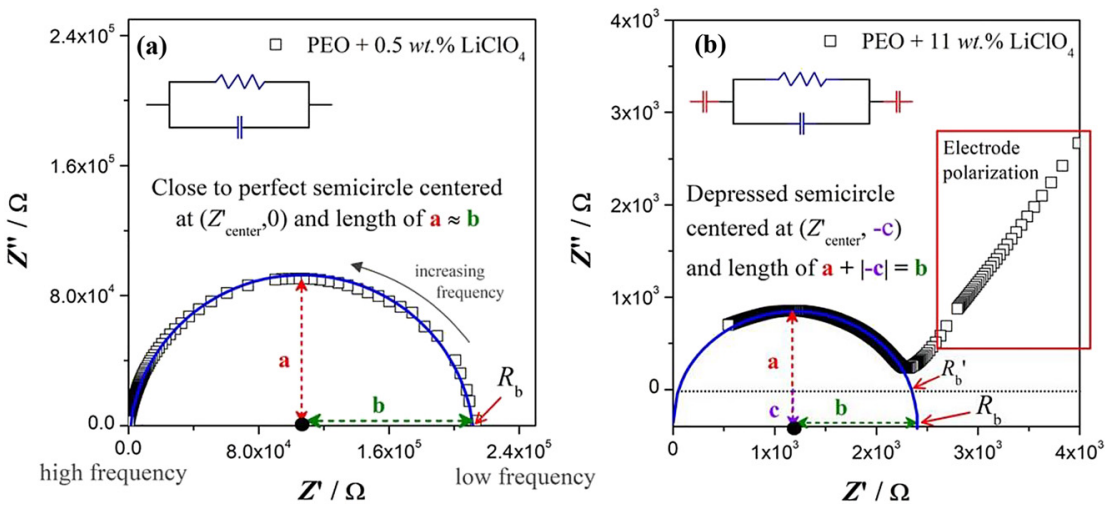

Figure 7: Nyquist plots for SPEs. (a) a close to perfect semicircle following Debye response, (b) a depressed semicircle with non-Debye response. The solid blue curve is the semicircle fitting of the impedance data estimated from Equation (3).

In this article, we also demonstrate a manual graphical approach, which can be adopted easily for estimation of $R_{\mathrm{b}}$ value even without the use of any commercial graphical software and computer. This approach is shown in detailed in Supplementary File S2 - A step-by-step guideline to estimate $R_{\mathrm{b}}$ using manual graphical approach. This may serve as an educational tool for a beginner to get a better insight on the estimation of $R_{\mathrm{b}}$ value before venturing into commercial graphical software (e.g., S1) or commercial EIS software for estimation of $R_{\mathrm{b}}$ value (e.g., S3). $R_{\mathrm{b}}$ is one of the electrical properties of an electrolyte that determines the total resistance of the medium across the electric circuit, that is also inversely proportional to the conductivity of the materials $\left(R_{\mathrm{b}} \propto 1 / \sigma_{\mathrm{DC}}\right)$.

Figure 7 shows the fitted Nyquist plots [from Equation (3)] with the shape of close to perfect semicircle [c.f. Figure 7(a)] and incomplete depressed semicircle at high frequency region with an inclined spike (due to electrode polarization) at low frequency region [c.f. Figure 7(b)]. For the Nyquist plot with a close to perfect semicircle, the $R_{\mathrm{b}}$ of the SPE can be estimated rather straight forward using manual graphical approach (procedures refer to $\mathbf{S 2}$ ) by finding the intersection point of the semicircle and $x$-axis at low frequency region [c.f. Figure 7(a)]. In some cases, a depressed semicircle is observed [c.f. Figure 7(b)]. The $R_{\mathrm{b}}$ estimation should be handled with extra attention because the center of the depressed semicircle lies somewhere below $Z^{\prime \prime}=0$. The $R_{\mathrm{b}}$ value should be estimated from the intersection point of the corrected centered semicircle at $Z^{\prime}$ axis. Figure $7(\mathrm{a})$ displays the close to perfect semicircle impedance data points that centered at $\left(Z_{\text {center }}^{\prime}, 0\right)$, where $Z_{\text {center }}^{\prime}=R_{\mathrm{b}} / 2$ and the radius of a should be equivalent to the radius of $\mathbf{b}$. Figure $7(\mathrm{~b})$ displays a depressed semicircle impedance data points that centered at $\left(Z_{\text {center }}^{\prime},-\mathbf{c}\right)$ with the radius of $(\mathrm{a}+|-\mathbf{c}|)$ is equivalent to the radius of $\mathbf{b}$. Frequently, the $R_{\mathrm{b}}$ value of depressed semicircle is estimated imprecisely from the intersection point of the semicircle at $Z^{\prime}$ axis that centered at $\left(Z^{\prime}{ }_{\text {center }}, 0\right)$, which we term here as $R_{\mathrm{b}}{ }^{\prime}$.

The estimation of $R_{\mathrm{b}}$ value may differ $>10 \%$ error if the estimation is done imprecisely. For instance, for $\mathrm{PEO}+11 \mathrm{wt}$. \% $\mathrm{LiClO}_{4}$ system, when a good estimation practice is followed, $R_{\mathrm{b}}$ value of $2.40 \times 10^{3} \Omega$ is obtained, otherwise $R_{\mathrm{b}}{ }^{\prime}$ value of $2.10 \times 10^{3} \Omega$ is recorded, where $>10 \%$ error is noted. It turns out that if $R_{\mathrm{b}}{ }^{\prime}$ values are used, we obtain $\sigma_{\mathrm{DC}}$ ' instead of $\sigma_{\mathrm{DC}}$ values.

Figure 8 illustrates the data interpretation using $R_{\mathrm{b}}$ and $R_{\mathrm{b}}{ }^{\prime}$ values, respectively for PEO-salt systems. Figure 8 depicts the double-logarithmic plots of conductivity $\left(\sigma_{\mathrm{DC}}\right)$ as a function of salt concentration $\left(Y_{\mathrm{S}}\right)$. Salt concentration $Y_{\mathrm{S}}=$ (mass of salt)/(mass of polymer). It turns out the plots denote a power law distribution due to the presence of the functional relationship between the quantities (Chan \& Kammer, 2008; Harun, Chan, \& Winie, 2017). The double-logarithmic plot of $\sigma_{\mathrm{DC}}$ versus $Y_{\mathrm{S}}$ can be read as

$$
\sigma_{\mathrm{DC}}=\sigma_{\mathrm{o}} Y_{\mathrm{s}}^{\mathrm{x}}
$$

which yields the quantity of $\sigma_{\mathrm{o}}$ and the slope of the plot can be expressed by power law exponent of $x$ (Chan \& Kammer, 2008; Harun et al., 2017). The exponent $x$ may be designated as the extent of interaction or correlation power between salt molecules and the polymer chains. We observed lower exponent $x$ values, $x=1.95$ using $R_{\mathrm{b}}$ values as compared to $x=2.13$ using $R_{\mathrm{b}}{ }^{\prime}$ values for PEO-salt system [c.f. Figure 8(a) and (b), respectively, where $R_{\mathrm{b}}{ }^{\prime}<R_{\mathrm{b}}$ ]. Similar observation for PMA. For systems display depressed semicircle of the Nyquist plots, higher 

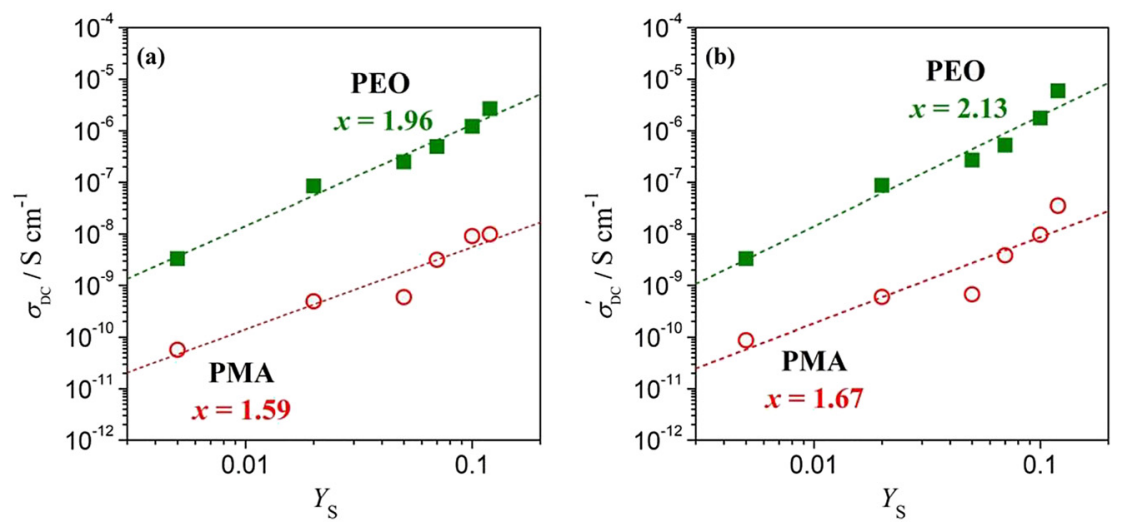

Figure 8: (a) $\sigma_{\mathrm{DC}}$ values estimated from $R_{\mathrm{b}}$ values and (b) imprecise $\sigma_{\mathrm{DC}}{ }^{\prime}$ values estimated from $R_{\mathrm{b}}$ ' values for (ם) PEO-salt and (O) PMA-salt systems. The dotted line represents the linear regressions after Equation (4).

Table 2: $R_{\mathrm{b}}$ and $\sigma_{\mathrm{DC}}$ values of PEO-LiClO ${ }_{4}$ estimated using different approaches.

\begin{tabular}{lrr}
\hline Approach used & PEO + 0.5 wt. \% of $\mathrm{LiClO}_{\mathbf{4}} \boldsymbol{R}_{\mathrm{b}}(\Omega)\left[\sigma_{\mathrm{DC}}\left(\mathbf{S} \mathbf{~ c m}^{-1}\right)\right]$ & PEO + 11 wt. \% of LiClO ${ }_{\mathbf{4}} \boldsymbol{R}_{\mathrm{b}}(\Omega)\left[\sigma_{\mathrm{DC}}\left(\mathbf{S} \mathbf{~ c m}^{-1}\right)\right]$ \\
\hline Manual graphical & $2.81 \times 10^{6}\left[3.32 \times 10^{-9}\right]$ & $2.41 \times 10^{3}\left[5.55 \times 10^{-6}\right]$ \\
Mathematical regression & $2.80 \times 10^{6}\left[3.33 \times 10^{-9}\right]$ & $2.40 \times 10^{3}\left[5.57 \times 10^{-6}\right]$ \\
ElS software (Nova, 2018) & $2.82 \times 10^{6}\left[3.31 \times 10^{-9}\right]$ & $2.49 \times 10^{3}\left[5.37 \times 10^{-6}\right]$ \\
\hline
\end{tabular}

conductivity $\left(\sigma_{\mathrm{DC}}{ }^{\prime}\right)$ values are estimated from $R_{\mathrm{b}}{ }^{\prime}$ values. This may lead to imprecise data interpretation subsequently. Hence, starting from the basic in estimation of $R_{\mathrm{b}}$ values is of important for beginners in order to interpret data precisely.

The approach for estimation of $R_{\mathrm{b}}$ value that we proposed using mathematical regression approach (refer to $\mathbf{S 1}$ ) and manual graphical approach (refer to $\mathbf{S 2}$ ) are easy to be followed by beginners. A comparison of the reproducibility of the $R_{\mathrm{b}}$ values estimated using (i) mathematical regression approach, (ii) manual graphical approach and (iii) a commercial EIS software [refer to $\mathbf{S} 3$ - A brief explanation on the estimation of $R_{\mathrm{b}}$ value using Nova software (version 2.1.4, Metrohm Autolab B.V., Utrecht, Netherlands)] is attempted as shown in Table 2. The reproducibility of $R_{\mathrm{b}}$ values using the three different approach can be observed.

The guides in $\mathbf{S 1}$ and $\mathbf{S} \mathbf{2}$ enable the users to understand the basic working principles for estimation of $R_{\mathrm{b}}$ when they rely on the EIS software in the subsequent attempt. We note here, different EIS software may have different mathematical algorithms for estimation of $R_{\mathrm{b}}$ value. With the basic understanding as presented in $\mathbf{S 1}$ and S2, the users may choose a suitable mathematical algorithms or suitable equivalent circuit for data extraction based on the shape of the semicircle.

Besides, a more detailed explanation on phenomenological dielectric response of SPEs and non-SPEs in the classic sense as mentioned herein will be discussed in the Part 2 of the next article. We will demonstrate the frequency-dependant impedance plots are the key quantities of the interest to decouple the short-range and long-range dielectric properties of the electrolytes and their interfaces with the conducting electrodes.

\section{Conclusion}

The basic working principles of electrolyte and electrochemical impedance spectroscopy were presented here for the delivery of insightful background for beginners or young researchers from undergraduate or postgraduate levels. This article covers the background of the electrolytes, instrumentation of EIS, step-by-step procedures to elucidate $R_{\mathrm{b}}$ values of electrolytes from various approaches provided with several examples and exercises, as well as a case study of data interpretation. Next, phenomenological dielectric response of SPEs and non-SPEs will be highlighted in Part 2 of next article. This insightful initiative can be served as the selflearning resources and coaching materials for educational purposes. 
Acknowledgments: The authors would like to express appreciation to Mdm. Amirah Hashifudin for providing impedance result of PEO with 0.5 wt. \% of LiClO4.

Author contributions: All the authors have accepted responsibility for the entire content of this submitted manuscript and approved submission.

Research funding: The authors would also like to express the greatest appreciation to Universiti Teknologi MARA (UiTM), Malaysia for granting LESTARI Grant (600-IRMI/MyRA/5/3/LESTARI (1072/2017)) and Ministry of Education, Malaysia for providing MyBrain15 (MyPhD) to financially support some experimental work of this article.

Conflict of interest statement: The authors declare no conflicts of interest regarding this article.

\section{References}

Abdul Halim, S. I., Chan, C. H., \& Kammer, H.-W. (2019). About glass transition in polymer-salt mixtures. Polymer Testing, 79, 105994.

Abdul Karim, S. R. B., Chan, C. H., \& Sim, L. H. (2017). Impedance spectroscopy: a practical guide to evaluate parameters of a Nyquist plot for solid polymer electrolyte applications. Functional Polymeric Composites: Macro to Nanoscales, 1st edition, 97-128. https://doi.org/10.1201/9781315207452-5.

Adar, R. M., Markovich, T., \& Andelman, D. (2017). Bjerrum pairs in ionic solutions: a Poisson-Boltzmann approach. Journal of Chemical Physics, 146(19), 1-8.

Baril, D., Michot, C., \& Armand, M. (1997). Electrochemistry of liquids versus solids: polymer electrolytes. Solid State lonics, 94(1-4), 35-47.

Baskoro, F., Wong, H. Q., \& Yen, H. J. (2019). Strategic structural design of a gel polymer electrolyte toward a high efficiency lithiumion battery. ACS Applied Energy Materials, 2(6), 3937-3971.

Chan, C. H., \& Kammer, H.-W. (2008). Properties of solid solutions of poly(ethylene oxide)/epoxidized natural rubber blends and LiCl04. Journal of Applied Polymer Science, 110, 424-432.

Chan, C. H., \& Kammer, H. W. (2015). Polymer electrolytes-relaxation and transport properties. Ionics, 21(4), 927-934.

Chan, C. H., \& Kammer, H. W. (2018). Characterization of polymer electrolytes by dielectric response using electrochemical impedance spectroscopy. Pure and Applied Chemistry, 90(6), 939-953.

Chan, C. H., \& Kammer, H. W. (2020). Low frequency dielectric relaxation and conductance of solid polymer electrolytes with PEO and blends of PEO and PMMA. Polymers, 12, 1-19.

Cole, K. S., \& Cole, R. H. (1941). Dispersion and absorption in dielectrics I. Alternating current characteristics. The Journal of Chemical Physics, 9(4), 341-351.

Debye, P. (1929). Polar molecules (p. 94). New York: Chemical Catalogue Company.

Debye, P., \& Falkenhagen, H. (1928). Dispersion of the conductivity and dielectric constants of strong electrolytes. Physikalische Zeitschrift, 29(121-132), 401-426.

Frumkin, A. (1940). Part II. - (A) Electrokinetic equations. The study of the double layer at the metal-solution interface by electrokinetic and electrochemical methods. Transactions of the Faraday Society, 35, 117-127.

Fuoss, R. M., Onsager, L., \& Skinner, J. F. (1965). The conductance of symmetrical electrolytes. V. The conductance equation. Journal of Physical Chemistry, 69(8), 2581-2594.

Grahame, D. C. (1947). The electrical double layer and the theory of electrocapillarity. Chemical Reviews, 41(3), 441-501.

Halim, S. I. A., Chan, C. H., \& Winie, T. (2017). Thermal, conductivity and molecular interaction studies of poly(ethylene oxide)/ poly(methyl acrylate) solid polymer electrolytes. Macromolecular Symposia, 371(1), 114-124.

Harun, F., Chan, C. H., \& Winie, T. (2017). Influence of molar mass on the thermal properties, conductivity and intermolecular interaction of poly(ethylene oxide) solid polymer electrolytes. Polymer International, 66(6), 830-838.

Hussin, N. S., Harun, F., \& Chan, C. H. (2017). Thermal roperties and conductivity of thermally treated epoxidized natural rubberbased solid polymer electrolytes. Macromolecular Symposia, 376(1), 1-7.

Klinklai, W., Kawahara, S., Marwanta, E., Mizumo, T., Isono, Y., \& Ohno, H. (2006). Ionic conductivity of highly deproteinized natural rubber having various amount of epoxy group mixed with lithium salt. Solid State lonics, 177(37-38), 3251-3257.

Kong, L., Li, C., Jiang, J., \& Pecht, M. G. (2018). Li-ion battery fire hazards and safety strategies. Energies, 11(9), 1-11.

Larson, C., Peele, B., Li, S., Robinson, S., Totaro, M., Beccai, L., ... Shephard, R. (2016). Highly stretchable electroluminescent skin for optical signaling and tactile sensing. Science, 351(6277), 1071-1074.

Learn Engineering. (2019). Lithium-ion battery, How does it work? Retrieved from https://www.youtube.com/watch? $\mathrm{v}=\mathrm{VxMM4g} 2 \mathrm{Sk} 8 \mathrm{U}$.

Lee, A. A., Perez-Martinez, C. S., Smith, A. M., \& Perkin, S. (2017). Underscreening in concentrated electrolytes. Faraday Discussions, 199, 239-259. 
Li, Q., Chen, J., Fan, L., Kong, X., \& Lu, Y. (2016). Progress in electrolytes for rechargeable Li-based batteries and beyond. Green Energy \& Environment, 1(1), 18-42.

Liu, J., Bao, Z., Cui, Y., Dufek, E. J., Goodenough, J. B., Khalifah, P., .. Meng, Y. S. (2019). Pathways for practical high-energy longcycling lithium metal batteries. Nature Energy, 4(3), 180-186.

Lopez, J., Mackanic, D. G., Cui, Y., \& Bao, Z. (2019). Designing polymers for advanced battery chemistries. Nature Reviews Materials, 4(5), 312-330.

Mackanic, D. G., Yan, X., Zhang, Q., Matsuhisa, N., Yu, Z., Jiang, Y., ... Chen, X. (2019). Decoupling of mechanical properties and ionic conductivity in supramolecular lithium ion conductors. Nature Communications, 10(1), 1-11.

Madani, S. S., Schaltz, E., \& Kaer, S. K. (2019). An electrical equivalent circuit model of a lithium titanate oxide battery. Batteries, 5(31), 1-14.

Manthiram, A., Yu, X., \& Wang, S. (2017). Lithium battery chemistries enabled by solid-state electrolytes. Nature Reviews Materials, 2(4), 1-16.

Miyamoto, A., Lee, S., Cooray, N. F., Lee, S., Mori, M., Matsuhisa, N., ... Someya, T. (2017). Inflammation-free, gas-permeable, lightweight, stretchable on-skin electronics with nanomeshes. Nature Nanotechnology, 12(9), 907-913.

Mohd Yusoff, S. N. H., Sim, L. H., Chan, C. H., Hashifudin, A., \& Kammer, H.-W. (2013). Solid solution of polymer electrolytes based on modified natural rubber. Polymer Research Journal, 7, 159-169.

Muthukumar, M. (2012). Polymer translocation. Boca Raton: CRC Press, Taylor \& Francis Group.

Ngai, K. S., Ramesh, S., Ramesh, K., \& Juan, J. C. (2016). A review of polymer electrolytes: fundamental, approaches and applications. Ionics, 22, 1259-1279.

Nova. (2018). Nova (Version 2.1.4) [Computer Software]. Utrecht, Netherlands: Metrohm Autolab B.V.

Pei, Z., Zhao, X., Yuan, H., Peng, Z., \& Wu, L. (2018). An equivalent circuit model for lithium battery of electric vehicle considering self-healing characteristic. Journal of Control Science and Engineering, 2018, 1-11.

Plante, G. (1884). Gaston plante. USA: Scientific American.

Pratap, R., \& Chandra, S. (2013). Ion transport studies in PEO: $\mathrm{NH}_{4} \mathrm{ClO}_{4}$ polymer electrolyte and its composite with $\mathrm{Al}_{2} \mathrm{O}_{3}$. Polymer Bulletin, 70(11), 3075-3089.

Raja, V., Sharma, A. K., \& Rao, V. V. R. N. (2004). Impedance spectroscopic and dielectric analysis of PMMA-CO-P4VPNO polymer films. Materials Letters, 58(26), 3242-3247.

Ramesh, S., \& Bing, K. N. (2012). Conductivity, mechanical and thermal studies on poly(methyl methacrylate)-based polymer electrolytes complexed with lithium tetraborate and propylene carbonate. Journal of Materials Engineering and Performance, 21(1), 89-94.

Ramström, 0. (2019). Scientific background on the nobel prize in chemistry 2019 - lithium-ion batteries. The Royal Swedish Academy of Sciences, 1, 1-13.

Randles, J. E. B. (1947). Kinetics of rapid electrode reactions. Discussions of the Faraday Society, 1, 11-19.

Stephan, A. M. (2006). Review on gel polymer electrolytes for lithium batteries. European Polymer Journal, 42(1), 21-42.

Su, Y.-L., Wang, J., \& Liu, H.-Z. (2002). Melt, hydration, and micellization of the PEO-PPO-PEO block copolymer studied by FTIR spectroscopy. Journal of Colloid and Interface Science, 251(2), 417-23.

Venkateswarlu, M., \& Satyanarayana, N. (1998). AC conductivity studies of silver based fast ion conducting glassy materials for solid state batteries. Materials Science and Engineering: B, 54(3), 189-195.

Voss, E. (1990). The Gaston planté medal address. Journal of Power Sources, 30, 7-10.

Zhang, Q. H., \& Chen, D. J. (2004). Percolation threshold and morphology of composites of conducting carbon black/ polypropylene/EVA. Journal of Materials Science, 39(5), 1751-1757.

Supplementary Material: The online version of this article offers supplementary material (https://doi.org/10.1515/cti-2020-0011). Three supplementary files (i.e., S1 - A step-by-step guideline to estimate bulk resistance $\left(R_{b}\right)$ using mathematical regression with commercial graphical software, $\mathbf{S 2}$ - A step-by-step guideline to estimate $R_{b}$ using manual graphical approach, supplemented with several exercises and S3 - A step-by-step guideline to estimate $R_{b}$ using exclusive EIS software) are provided separately for educational, practical, and self-learning purposes. 